\title{
Low Temperature Transport Properties of Strongly Interacting Systems - Thermal Conductivity of Spin-1/2 Chains
}

\section{Dedicated to Nando Mancini on the occasion of his 60th birthday}

\author{
N. Andrei \\ Center for Materials Theory, Rutgers University, Piscataway, NJ 08854-8019
}

\author{
E. Shimshoni \\ Department of Mathematics-Physics, University of Haifa at Oranim, Tivon 36006, Israel
}

\author{
A. Rosch \\ Institut für Theorie der Kondensierten Materie, Universität Karlsruhe, D-76128 Karlsruhe, Germany
}

\begin{abstract}
We outline a general approach to the computation of transport properties of interacting systems at low temperetures and frequencies. We show that if the fixed point and the irrelevant operators around it are known, then by studying the structure of the softly violated conserved currents chracterizing the fixed point one may set up an effective calculation in terms of a memory matrix formalism. We apply this approach to the computation of thermal conductivity of spin chains embedded in a matter matrix and interacting with its phonons. The results are found to be in very good agreement with experiment.
\end{abstract}

The study of transport properties of strongly interacting systems has been of great theortical and experimental interest for a long time. We shall concentrate in this contribution on developing an effective approach to the problem when the system is not far from its fixed point; in other words, when it is probed at low temperatures and at low frequencies. Subsequently we shall carry it out in detail for a system of spin $1 / 2$ chains coupled to phonons.

Our approach consists of the following elements:

1. Identify the fixed point of the hamiltonian which describes the system, as well as the irrelevant operators around it.

The fixed point itself is typically insufficient to describe low energy transport properties; it is scale invariant and translationally invariant and thus unable to degrade a current, leading to infinite conductivity. To obtain finite conductivity one needs to take into account terms which break translational invariance and, more generally, violate the conservation laws associated with the fixed point. 2. Study the (weakly violated) conserved charges around the fixed point hamiltonian.

A fixed point $H^{*}$ is scale invariant and often has several conserved quantities $P,\left[P, H^{*}\right]=0$, associated with it. In 1-d, for example, if the fixed point is conformally invariant it has an infinite number of conserved quantities. When the irrelevant operators around the fixed point are taken into account, most of these quantities no longer commute with the low energy hamiltonian. The conservation of most of these quantites is strongly violated, but some may be only weakly violated and then significantly influence the low energy dynamics of the system.

Typically the current whose correlations determining the transport properties under considerations will be among those almost conserved charges, or "protected" by them in the following sense. When a system possesses some conserved quantities $P$, these may "protect" the cur- rent $J$ from degrading (this occurs when the crosssusceptibility $\chi_{J P} \neq 0$ ) leading to a pure (i.e. $\delta(\omega)$ ) Drude peak and infinite d.c. conductivity. When the conservation of the pseudo-momenta $P$ is softly violated they will, instead, lead to very long time tails in the decay of the current $J$. This occurs since states with a finite pseudo-momentum $P$ typically carry also a finite current $J$ since $\chi_{J P} \neq 0$. The component of the current "parallel" to $P, J_{\| P}=\left(\chi_{P J} / \chi_{P P}\right) P$ will therefore decay slowly. The presence of such approximately conserved quantities leads then to a natural hydrodynamic description of the system where a separation of fast and slowly decaying modes takes place and a consistent scheme of calculation of the slow mode conductivities can be carried out in terms of matrix of decay rates of these modes.

Let us explain and illustrate these ideas in more detail. We begin by arguing that given a conserved charge $P$, it will "protect" $J$ if $\chi_{P J} \neq 0$. Indeed, imagine preparing at $t=0$ a state carrying a current $\langle J\rangle$. Then necessarily that state will also have a non vanishing $\langle P\rangle$,

$$
\langle P\rangle=\frac{\chi_{P J}}{\chi_{J J}}\langle J\rangle .
$$

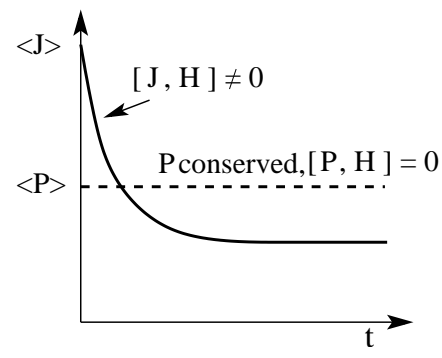

FIG. 1

In the limit $t \rightarrow \infty$ since $P$ is conserved its expectation value will not have changed, and it will in its turn induce 
a non vanishing expectation value for $J$,

$$
\lim _{t \rightarrow \infty}\langle J\rangle=\frac{\chi_{J P}}{\chi_{P P}}\langle P\rangle=\frac{\chi_{J P}^{2}}{\chi_{P P} \chi_{J J}}\langle J(t=0)\rangle
$$

(see Fig. 1). Since the current tends asymptotically to a constant value we find that the conductivity will have a $\delta(\omega)$-Drude peak containing a fraction $\frac{\chi_{J P}^{2}}{\chi_{P P} \chi_{J J}}$ of the total weight; in other words, a Drude weight $D=\frac{1}{2} \frac{\chi_{J P}^{2}}{\chi_{P P}}$. An immediate consequence is that integrable models, having infinitely many conserved quantities, will typically also have an infinite dc conductivity.

If the charge $P$ is not conserved but slowly decaying it will induce slow (long-time) decay in $J$ (see Fig. 2).

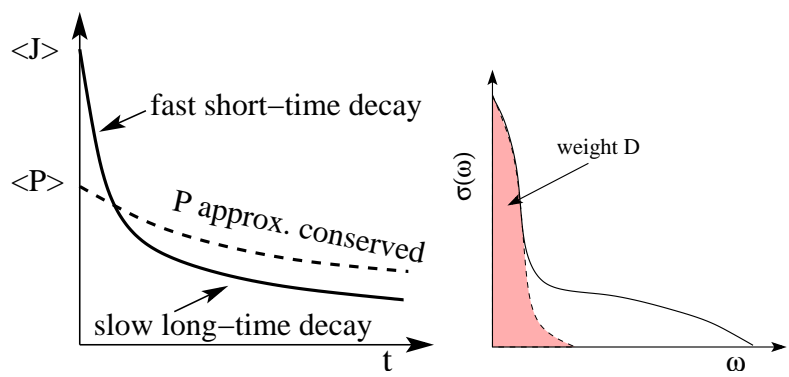

FIG. 2

This slow decay will show up as a peak in $\sigma(\omega)$ and the decay-rate $\Gamma_{P}$ of $P$ will determine the width of the peak and the dc value $\sigma(0) \approx D / \Gamma_{P}$ of the optical conductivity, the area under the peak being again, $D=\frac{1}{2} \frac{\chi_{. J P}^{2}}{\chi_{P P}}$.

3. Having identified the slowly decaying charges of the effective model describing the low energy properties of the system we can compute the low temperature dc transport using a method that separates fast and slow modes, incorporating the former in the dynamics of the latter. Such a method is the memory matrix approach which can be used very efficiently and controllably when combined with the RG considerations outlined above.

Let us apply this approach to a system of spin chains embedded in some 3-d lattice and interacting with its phonons. Such systems (including, in particular, various compounds of $\mathrm{SrCuO}$ ) have been recently studied in detail by, e.g., Sologubenko et al $\stackrel{1}{\frac{1}{1}}$ The authors have measured the heat conductivity along the three main axes of the sample. They observed that while conductivities along the $a$ and $c$ axes almost coincide, the conductivity along $b$, the axis along which the spin chains lie, has an enhancement which they interpret as being due to contribution of the spin degrees of freedom (Fig. 3, for example, presents data from a corresponding measurement in $\mathrm{Sr}_{2} \mathrm{CuO}_{3}$ ). In the temperature range $60 \mathrm{~K} \leq T \leq 200 \mathrm{~K}$, they gave the fit:

$$
\begin{aligned}
\kappa_{s}(T) & \sim \exp \left(T^{*} / T\right) \\
T^{*} & \approx 0.42 \Theta_{D}
\end{aligned}
$$

where $\kappa_{s}$ is the spin contribution obtained after subtracting the phonon background from $\kappa_{b}$. The relevant energy scales of the system are: The Debye temperature characterizing the phonons $-\Theta_{D} \sim 400 \mathrm{~K}$ and the spinon interaction scale $J / k_{B} \sim 2600 \mathrm{~K}$ characterizing the spin chain.

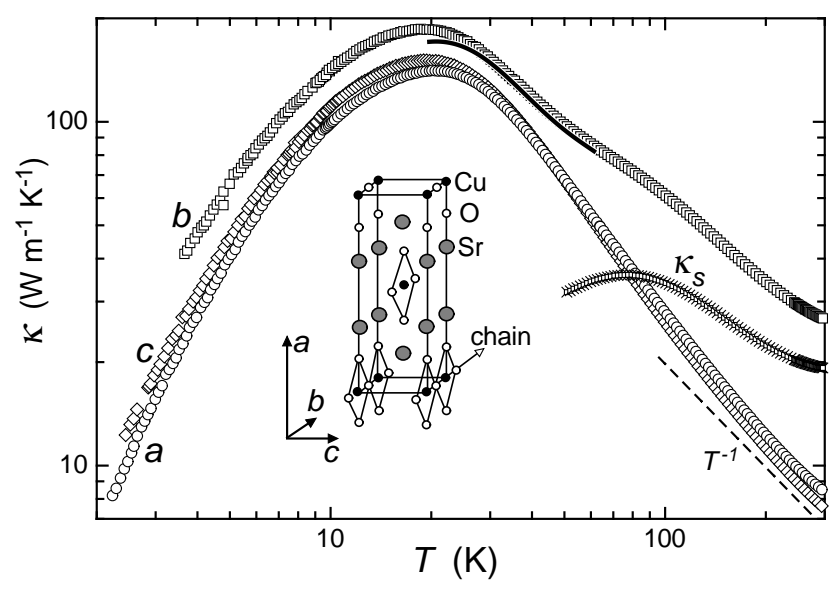

FIG. 3

One immediate question that arises is why is $\kappa_{s}$ actually determined by the lower scale $\Theta_{D}$. Furthermore, by plotting the above data of $\ln \kappa_{s}$ and of the pure phonon contribution $\ln \kappa_{a, c}$ vs. $1 / T$, one observes that the slope of the latter is larger by a factor of 2 . Again, why?

We shall find that a rather subtle interplay of (approximate) conservation laws and quantum dynamics underlies the experimentally observed heat conductivity, and the approach outlined above is necessary to fully account for it.

We begin by discussing the low energy effective hamiltonian. First consider a single spin chain,

$$
H_{s}=\frac{1}{2} \sum_{i, j=1}^{N} J_{i j}\left(S_{i}^{+} S_{j}^{-}+S_{i}^{-} S_{j}^{+}\right)+\sum_{i, j=1}^{N} J_{i j}^{z} S_{i}^{z} S_{j}^{z}
$$

As is well known,$\frac{2}{2}$ for spin chains with short range interactions the fixed point hamiltonian is the Luttinger liquid,

$H_{L L}=-i(J a) \int d x\left(\psi_{R}^{\dagger} \partial_{x} \psi_{R}-\psi_{L}^{\dagger} \partial_{x} \psi_{L}\right)+J_{z} \int d x \rho(x)^{2}$

with $\psi_{R / L}$ being right/left moving fermi field, and $J$ and $J_{z}$ are some average values of $J_{i j}$ and $J_{i j}^{z}$ respectively. As a reminder, the fixed point can be obtained by carrying out a Wigner-Jordan tranformation and then linearizing the resulting fermions around the Fermi points $\pm k_{F}$ (see Fig. 4). 


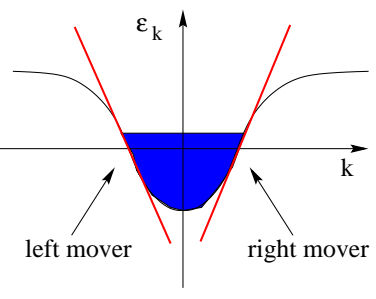

FIG. 4

It will be convenient to change to bosonic variables in terms of which the fixed point hamiltonian takes the form (to leading order in $\left|J_{z}\right| / J$ ),

$$
H_{L L}=v \int \frac{d x}{2 \pi}\left(K(\pi \Pi)^{2}+\frac{1}{K}\left(\partial_{x} \phi\right)^{2}\right)
$$

where

$$
v \approx\left(J+\frac{J_{z}}{\pi}\right) a, \quad K \approx \frac{1}{1+\frac{2 J_{z}}{\pi J}} .
$$

We now consider the irrelevant operators around the fixed point. The irrelevant operators come from various sources. We shall divide them to Umklapp and nonumklapp operators. To the latter category belong local terms coming from band curvature around $k_{F}$ : for example $\int \psi_{R}^{\dagger} \partial^{2} \psi_{R}$. However, the operators that are important for transport are the Umklapp operators that reflect the underlying lattice structure. Only they break translation invariance and can degrade the currents. They have the following structure:

$$
\begin{aligned}
H^{U} & =\sum_{n m} H_{n m}^{U} \\
H_{n m}^{U} & =g_{n m}^{U} \int d x\left[e^{i \Delta k_{n m} x} \prod_{j=0}^{n} \psi_{R}^{\dagger}(x+j a) \psi_{L}(x+j a)+h . c .\right] \\
& =\frac{g_{n m}^{U}}{(2 \pi a)^{n}} \int d x\left[e^{i \Delta k_{n m} x} e^{i 2 n \phi(x)}+\text { h.c. }\right]
\end{aligned}
$$

where $\Delta k_{n m}=n 2 k_{F}-m G$ is the momentum transfer associated with the Umklapp process where $n$ particles are transferred from one Fermi point to another while giving up to the lattice $m$ units of lattice momentum $G=2 \pi / a$. The particular values of the couplings $g_{n m}^{U}$ depend on the couplings in the microscopic model. These terms are irrelevant perturbations around the fixed point not only by power counting but also because the $x$-dependent exponentials in them suppress their contribution exponentially. However they are the only source of dissipation.

We now consider the complete system consisting of an array of parallel spin chains interacting with 3-dimensional acoustic phonons. The 3 -d phonon system projected along the axis describing deformations of the lattice parallel to the chains is described by

$$
H_{p}^{3 D}=\int \frac{d^{3} x}{2 \pi}\left[(\pi P)^{2}+\sum_{\mu} v_{\mu}^{2}\left(\partial_{\mu} q\right)^{2}\right],
$$

with $q$ the lattice deformation parallel to the spin chains direction, and $P$ the conjugate momentum.

Integrating the corresponding propagator over the perpendicular directions, we obtain the propagator along the chains:

$$
\int d^{2} k_{\perp} \frac{1}{\left(\omega^{2}+\sum_{\mu} v_{\mu}^{2} k_{\mu}^{2}\right)} \sim \ln \left[\left(\omega^{2}+v_{p}^{2} k^{2}\right) / \Theta_{D}^{2}\right] .
$$

In real space it takes the form $1 /\left(x^{2}+v_{p}^{2} t^{2}\right)$ with $v_{p}$ being the slowest phonon velocity. This again is the propagator of a $K=1$ Luttinger liquid describing the phonons, $H_{p}=v_{p} \int \frac{d x}{2 \pi}\left((\pi \Pi)^{2}+\left(\partial_{x} q\right)^{2}\right)$. Again we need to add to the combined phonon-spinon fixed point $H^{*}=\sum_{\alpha} H_{p}^{\alpha}+\sum_{\alpha} H_{L L}^{\alpha}$ (the summation $\alpha$ is over the spin chains) all the irrelevant operators. As before they fall into two categories, the Umklapp and non Umklapp operators. The most important ones of the former one have the form,

$$
H_{n m}^{U, s-p}=\frac{g_{n m}^{U, p}}{(2 \pi a)^{n}} \int d x\left[e^{i \Delta k_{n m} x} e^{i 2 n \phi} \partial_{x} q+\text { h.c. }\right]
$$

(where $\phi$ is the bosonic field in a particular chain), while an example of the latter is $H_{s, p}^{\text {nonU }}=\int(\partial \phi)^{2} \partial_{x} q$.

We now turn to our main interest, computing the thermal conductivity $\kappa_{s}(\omega, T)$ of the spin chains coupled to phonons. The thermal conductivity can be expressed in terms of a heat current correlation function,

$$
\kappa(\omega, T)=\left\langle J_{Q}, J_{Q}\right\rangle(\omega, T) / \omega
$$

where $J_{Q}$, the heat current expressed in bosonic variables, is $J_{Q}=-\sum_{\alpha} \int d x v^{2} \Pi_{\alpha} \partial_{x} \phi_{\alpha}-\int d^{3} x v_{p}^{2} P \partial_{x} q$. The correlation is to be computed with respect to the low-energy hamiltonian,

$$
H_{\text {low }-E}=H^{*}+H^{U}+H^{\text {nonU }} .
$$

As explained in the outline we need to identify the (approximately) conserved "charges" of the low-E Hamiltonian, to find out whether they induce a slow decay of the heat current. We now show that the quantities,

$$
\begin{aligned}
J_{s} & =v K \sum_{\alpha} \int d x\left[\psi_{R \alpha}^{\dagger} \psi_{R \alpha}-\psi_{L \alpha}^{\dagger} \psi_{L \alpha}\right]=v K \sum_{\alpha} \int d x \Pi_{\alpha} \\
P_{T} & =-\sum_{\alpha} \int d x \Pi_{\alpha} \partial_{x} \phi_{\alpha}-\int d^{3} x P \partial_{x} q
\end{aligned}
$$

where, $J_{s}$ is the spin current and $P_{T}$ the momentum operator, are the "slow modes" which in turn protect $J_{Q}$ rendering it slow too.

Indeed, $J_{s}$ and $P_{T}$ commute with $H_{L L}$ and with $H^{\text {non } U}$ and their conservation is violated only through $H^{U}$ with which they do not commute, thus inducing a slow current decay. More importantly, certain linear combinations of $J_{s}$ and $P_{T}$, the "pseudo-momenta"

$$
P_{n m}=\frac{1}{2 n} \Delta k_{n, m} J_{s}+P_{T}
$$


decay even slower as they commute with $H_{L L}+H^{\text {nonU }}+$ $H_{n m}^{U}+H_{n m}^{U, s-p}$ and are therefore exactly conserved if only a single type of Umklapp with quantum numbers $n$ and $m$ is present. We note that the pseudo momenta can be written as $P_{n m}=P_{l a t}+\frac{m}{2 n} G\left(N_{R}-N_{L}\right)$ with $P_{\text {lat }}=\sum_{k} k c_{k}^{\dagger} c_{k} \approx k_{F}\left(N_{R}-N_{L}\right)+P_{T}$ being the lattice momentum. Unlike other (approximately) conserved quantities, they decay exponentially slowly with the temperature as their violation requires processes away from the Fermi energy $\underline{3}$

The heat current on the other hand does not commute with both $H^{\text {nonU }}$ and $H^{U}$ and would therefore decay fast, but is protected by $J_{s}, P_{T}$ and their linear combinations since $\chi_{J_{Q}, J_{s}}, \chi_{J_{Q}, P_{T}} \neq 0$.

We proceed to discuss transport in the presence of several approximately conserved - "slow" - variables: $J_{1}, J_{2} \ldots J_{N}$. We shall introduce the memory matrix formalism ${ }^{4}$ which is very effective under the circumstances as it allows the separation of the slow modes $\left(J_{s}, P_{T}, J_{Q}\right.$, in our particular case) from the fast modes.

To set up the formalism one introduces a scalar product in the space of operators of the theory

$$
(A(t) \mid B) \equiv \frac{1}{\beta} \int_{0}^{\beta} d \lambda\left\langle A(t)^{\dagger} B(i \lambda)\right\rangle .
$$

In terms of this scalar product one can express the dynamic correlation functions as follows,

$$
\begin{aligned}
C_{A B}(\omega) & =\int_{0}^{\infty} d t e^{i \omega t}(A(t) \mid B) \\
& =\left(A\left|\frac{i}{\omega-\mathcal{L}}\right| B\right) \\
& =\frac{i T}{\omega} \int_{0}^{\infty} d t e^{i \omega t}\langle[A(t), B]\rangle-\frac{(A \mid B)}{i \omega}
\end{aligned}
$$

where the Liouville operator $\mathcal{L}$ is defined as $\mathcal{L} A=[H, A]$. The matrix of conductivities (Kubo formula) is then,

$$
\hat{\sigma}_{p q}(\omega, T)=\frac{1}{T V} C_{J_{p} J_{q}}(\omega)
$$

$(p, q=1 \cdots N)$. In our case the thermal conductivity is

$$
\kappa_{s}(\omega, T)=\frac{1}{T} \sigma_{Q Q}(\omega, T) .
$$

However the dc conductivity has no good perturbative expansion: $\sigma \sim 1 / \Gamma$, with $\Gamma$ the decay rate of the current is singular in perturbation theory. Put in other words, if we wish to compute the conductivity as a perturbative expansion of the irrelevant operators around the fixed point, this would be an arduous task in view of the fact that the conductivity computed from the fixed point is infinite. Furthermore, even the perturbative expansion for $1 / \sigma(\omega=0)$ turns out to be ill behaved in the presence of slow modes. While the short-time decay rate (see Fig. 2) of the current is perturbative, the $\omega \rightarrow 0$ limit requires to take into account the presence of approximate conservation laws.

We seek therefore to compute a quantity which has a good perturbative expansion, and to this purpose introduce $\hat{M}(\omega, T)$ - the Memory Matrix, essentially the matrix of relaxation rates.

The matrix is defined as

$$
\hat{M}_{p q}(\omega)=\frac{1}{T}\left(\partial_{t} J_{p}\left|\mathcal{Q} \frac{i}{\omega-\mathcal{Q L} \mathcal{Q}} \mathcal{Q}\right| \partial_{t} J_{q}\right)
$$

where $\mathcal{Q}$ is the projection away from slow modes

$$
\left.\mathcal{Q}=1-\sum_{p q} \mid J_{p}\right) \frac{1}{T}\left(\hat{\chi}^{-1}\right)_{p q}\left(J_{q} \mid .\right.
$$

In terms of the memory matrix the conductivity matrix is,

$$
\hat{\sigma}(\omega, T)=\hat{\chi}(T)(\hat{M}(\omega, T)-i \omega \hat{\chi}(T))^{-1} \hat{\chi}(T)
$$

with $\hat{\chi}$ the susceptibility matrix,

$$
\hat{\chi}_{p q}=\frac{1}{T V}\left(J_{p} \mid J_{q}\right) .
$$

Applying the formalism in our case we find that the memory matrix is a sum over the Umklapp processes $(n m)$, given - to leading order in $g_{m n}^{U}$ - by,

$$
\hat{M}=\frac{1}{T}\left[\sum_{n m}\left(\hat{M}_{n m}+\hat{M}_{n m, s-p}\right)\right]
$$

where (the matrix indices $p, q$ take the values $s, T, Q$ )

$$
\begin{aligned}
M_{n m}^{p q} & \equiv \frac{\left\langle F^{p} ; F^{q}\right\rangle_{\omega}^{0}-\left\langle F^{p} ; F^{q}\right\rangle_{\omega=0}^{0}}{i \omega}, \\
M_{n m, s-p}^{p q} & \equiv \frac{\left\langle F_{s-p}^{p} ; F_{s-p}^{q}\right\rangle_{\omega}^{0}-\left\langle F_{s-p}^{p} ; F_{s-p}^{q}\right\rangle_{\omega=0}^{0}}{i \omega} ;
\end{aligned}
$$

here $F^{p}=i\left[J_{p}, H^{U}\right], F_{s-p}^{p}=i\left[J_{p}, H^{U, s-p}\right]$ and $\left\langle F^{p} ; F^{q}\right\rangle_{\omega}^{0}$ is the retarded correlation function calculated with respect to $H^{*}$. As the perturbative expansion is in irrelavant operators with respect to the fixed point the expansion is expected to be rapidly converging at low temperatures if slow and fast time scales are well seperated and all of the slowest modes have been taken into account.

Carrying out the computation of the various correlation functions (see Ref. [5] for details) we have:

$$
\kappa_{s}(T) \approx v^{2} T^{3}\left[\left(\hat{M}^{-1}\right)_{T T}+2\left(\hat{M}^{-1}\right)_{Q T}+\left(\hat{M}^{-1}\right)_{Q Q}\right]
$$

with the typical matrix elements,

$$
\begin{aligned}
M_{n m}^{p q} & \sim\left(\Delta k_{n m}\right)^{\left(n^{2} K-2\right)} e^{-v \Delta k_{n m} / 2 T} \\
M_{n m, s-p}^{p q} & \sim T^{\left(2 n^{2} K-1\right)} e^{-v_{p} \Delta k_{n m} / 2 T} .
\end{aligned}
$$


Note that the spinon processes decay exponentially fast, with the exponent $-v \Delta k_{n m} / 2 T$, while the spinonphonon exponents contain the much slower phonon velocity $v_{p} \ll v$, leading to a much slower decay with the exponent $-v_{p} \Delta k_{n m} / 2 T$. The latter will therefore clearly determine the thermal conductivity.

But which of the scattering processes $(n, m)$ will dominate?

At low- $\mathrm{T}$ exponential factor prevails hence the smallest $\Delta k_{n m}$. At this point our discussion must distinguish between commensurate and incommensurate magnetization, or in fermionic language commensurate and incommensurate filling. The magnetization can be in principle tuned by varying an external magnetic field $h$.

Close to commensurate filling $k_{F} \approx G \frac{m_{0}}{2 n_{0}}$, and then the dominant processes appear to be $H_{n_{0} m_{0}}^{U}$ where $\Delta k_{n_{0}, m_{o}} \approx 0$. However, because of the conservation laws discussed earlier, it is the next leading term $H_{n_{1} m_{1}}^{U}$ with $\Delta k_{n_{1}, m_{1}}= \pm G / n_{0}$ which determines the decay rate. Technically, this arises as one of the eigenvalues of the matrix $\hat{M}$ is not affected by $H_{n_{0} m_{0}}^{U}$ (as $\left[P_{n_{0} m_{0}}, H_{n_{0} m_{0}}^{U}\right]=$ $0)$ but determined by $H_{n_{1} m_{1}}^{U}$. This smallest eigenvalue will then determine the size of $\hat{M}^{-1}$ and therefore the heat conductivity. The particular case of half filling is discussed in detail below.

On the other hand, at a typical incommensurate filling it will depend on the temperature which processes are dominant and subdominant and we need to sum over all terms (do saddle-point approximation with respect to $n_{1}$ ) and find,

$$
\kappa_{\text {typical }} \sim \exp \left[c(\beta v G)^{2 / 3}\right] .
$$

with $c$ a constant of order 1 .

We now turn to the experiment by Sologubenko et al. discussed earlier. The experiment was carried out at $h=0$ corresponding to half filling, $k_{F}=\pi / 2 a=G / 4$. Therefore $\Delta k_{21}=0 \quad(\mathrm{n}=2, \mathrm{~m}=1)$. But we need at least two Umplapp terms and the next smallest is $\Delta k=$ $G / 2,(n=1, m=0)$. Recall also that as $v_{p} \ll v$, it follows that $\hat{M}_{n m, s-p} \gg \hat{M}_{n m}$. Hence, the dominant contribution to the thermal conductivity comes from, $\left(\hat{M}^{-1}\right)_{T T} \approx 1 / M_{n=1, s-p}^{T T}(G / 2, T)$ and we have

$$
\kappa(T) \approx \kappa_{0}\left(T / T^{*}\right)^{2(1-K)} e^{T^{*} / T}
$$

with

$$
T^{*}=v_{p} G / 4 .
$$

We find therefore that the second strongest rate wins (cf. the expression for $\hat{M}$ ): it is determined by $v_{p}$ via a phonon process and is characterized by the momentum $G / 2$. The $G / 2$ transfer momentum characterizes the dominant spinon - phonon Umklapp process, and clearly distiguishes it from pure phonon Umklapp processes characterized by momentum transfer $G$. We expect therefore that in the pure phonon thermal conductivity (axes $a$ and $c$ in Fig. 3) the scale $2 T^{*}$ would appear.

To compare our findings with the experimental data we need to express our expression in terms of $\Theta_{D}$. Assuming an isotropic phonon dispersion one has: $\Theta_{D} \approx$ $v_{p}\left(6 \pi^{2} / a^{3}\right)^{1 / 3} \approx 0.6 v_{p} G$.

Therefore:

$$
\begin{aligned}
& T^{*} \approx 0.4 \Theta_{D}, \text { (theory) } \\
& T^{*} \approx 0.42 \Theta_{D} \text {, (experiment) } .
\end{aligned}
$$

Taking into account possible ambiguities in the fits to the experiments and that the phonon dispersion is probably not completely isotropic, part of this excellent agreement may be accidential. But further confirmation of our theory comes from the observation that the ratio of slopes of the spinon contribution compared to the pure phonon contribution (on a semilogarithmic graph of $\kappa$ vs. $1 / T$ see Fig. $5 \underline{\underline{\underline{6}}}$ ) is approximately $1: 2$ as discussed earlier.

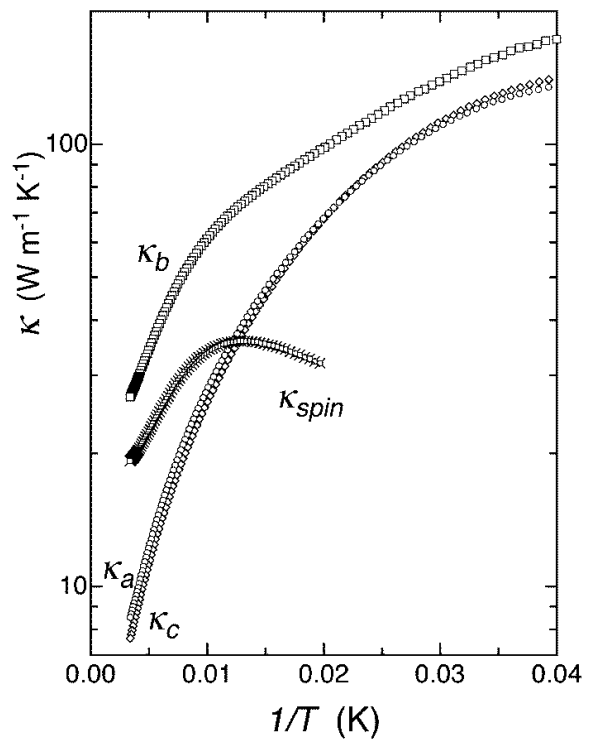

FIG. 5

We may also consider the effect of a magnetic field on the thermal conductivity. (To observe it experimentally one needs a material with much smaller spin energy scales $J$ than $\mathrm{SrCuO}$ ). The magnetic field modifies the value of $k_{F}$ according to

$$
k_{F}=\frac{\pi}{2 a}(1+M) \approx \frac{\pi}{2 a}(1+h /(\pi J)) .
$$

As the field $h$ is varied the system passes through commensurate fillings, $\Delta k_{n m}=n 2 k_{F}-m G=0$ and incommensurate fillings, $\Delta k_{n m}=n 2 k_{F}-m G \neq 0$. Thus different Umklapp operators become effective leading to a fractal-like dependence on $M \approx h / \pi J$ (see Fig. 6). 


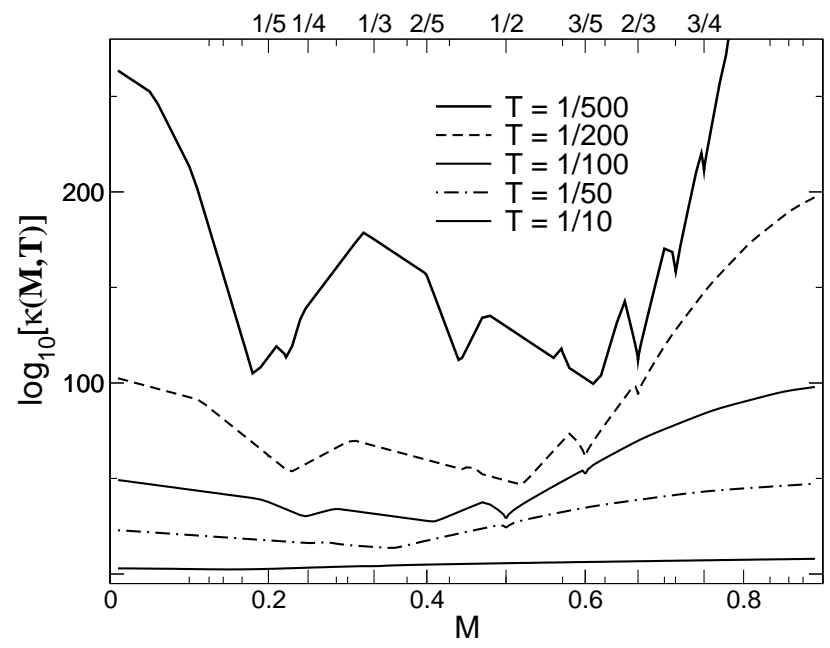

FIG. 6
We expect some dips to be experimentally observable.

In conclusion,

- Transport is strongly influenced by conserved "charges": low energy processes cannot relax heat current.

- Exponents are determined by slowest mode in the system: typically phonons.

- Memory Matrix approach, separating slow and fast modes, allows controllable calculations.

- Calculations fit experiments

- Interesting predictions on the magnetization dependence of the heat transport have been made.
1 A. V. Sologubenko et al., Phys. Rev. B 62, R6108 (2000).

${ }^{2}$ I. Affleck, in Fields, Strings and Critical Phenomena, Les Houches, Session XLIX, edited by E. Brezin and J. ZinnJustin (North-Holland, Amsterdam, 1988).

3 A. Rosch and N. Andrei, Phys. Rev. Lett. 85, 1092 (2000); A. Rosch and N. Andrei, Journal Low Temp Phys. 126, 1195 (2002).

${ }^{4}$ D. Forster, Hydrodynamic Fluctuations, Broken Symme- try, and Correlation Functions, (Benjamin, Massachusetts, 1975).

5 E. Shimshoni, N. Andrei and A. Rosch, to be published in Phys. Rev. B (condmat/0304641).

${ }^{6}$ We thank Alex Sologubenko for providing us with the replotted data. 